\title{
Direct Estimation of the Reynolds Stress Vertical Structure in the Nearshore
}

\author{
FALK FedDERSEN \\ Scripps Institution of Oceanography, La Jolla, California \\ A. J. Williams III \\ Woods Hole Oceanographic Institution, Woods Hole, Massachusetts
}

(Manuscript received 31 May 2005, in final form 13 April 2006)

\begin{abstract}
Measurements of the vertical Reynolds stress components in the wave-dominated nearshore are required to diagnose momentum and turbulence dynamics. Removing wave bias from Reynolds stress estimates is critical to a successful diagnosis. Here two existing Reynolds stress estimation methods (those of Trowbridge, and Shaw and Trowbridge) for wave-dominated environments and an extended method (FW) that is a combination of the two are tested with a vertical array of three current meters deployed in 3.2-m water depth off an ocean beach. During the 175-h-long experiment the instruments were seaward of the surfzone and the alongshore current was wind driven. Intercomparison of Reynolds stress methods reveals that the Trowbridge method is wave bias dominated. Tests of the integrated cospectra are used to reject bad Reynolds stress estimates, and the Shaw and Trowbridge estimates are rejected more often than FW estimates. With the FW method, wave bias remains apparent in the cross-shore component of the Reynolds stress. However, the alongshore component of Reynolds stress measured at the three current meters are related to each other with a vertically uniform first EOF containing $73 \%$ of the variance, indicating the presence of a constant stress layer. This is the first time the vertical structure of Reynolds stress has been measured in a wave-dominated environment. The Reynolds stress is, albeit weakly, related to the wind stress and a parameterized bottom stress. Using derived wave bias and bottom stress parameterizations, the effect of wave bias on Reynolds stress estimates is shown to be weaker for more typical surfzone conditions (with both stronger waves and currents than those observed here).
\end{abstract}

\section{Introduction}

The Reynolds stress terms $\overline{u^{\prime} w^{\prime}}$ and $\overline{v^{\prime} w^{\prime}}$, where $u^{\prime}$, $v^{\prime}$, and $w^{\prime}$, respectively, represent the cross-shore, alongshore, and vertical components of turbulent velocities and the overbar is an averaging operator, play an important role in the mean momentum and turbulence dynamics of boundary layer flows. Estimating the Reynolds stress from observations is crucial in diagnosing these dynamics. Often the Reynolds decomposition between mean and turbulent velocity components and its application to observations is straightforward. However, in coastal and nearshore regions, energetic surface gravity waves complicate the situation by introducing

Corresponding author address: Falk Feddersen, Integrative Oceanography Division, Scripps Institution of Oceanography, University of California, San Diego, La Jolla, CA 92093-0209.

E-mail: falk@coast.ucsd.edu wave biases that contaminate the Reynolds stress signal (e.g., Grant and Madsen 1986; Trowbridge 1998, hereafter T98; Shaw and Trowbridge 2001, hereafter ST01).

The first practical Reynolds stress estimation method in the presence of orbital wave motions involves differencing velocity observations from adjacent sensors (T98). If the sensor separation is small relative to a typical wavelength, but long relative to the turbulence length scale, then the differenced velocity contains only turbulent components. Basically, the wave velocities at the two sensors are assumed equal and thus are canceled by differencing. The covariance of the differenced alongshore and vertical velocities gives the Reynolds stress term $\overline{v^{\prime} w^{\prime}}$. T98 applied this method to horizontally separated observations in 6-m depth off of Duck, North Carolina (Trowbridge and Agrawal 1995). This method was subsequently used with horizontally separated sensors $1 \mathrm{~m}$ above the bed in $4.5-\mathrm{m}$ water depth off of Duck to estimate $\overline{v^{\prime} w^{\prime}}$, resulting in a successful

DOI: 10.1175/JTECH1953.1 
diagnosis of the alongshore momentum and turbulence dynamics (Trowbridge and Elgar 2001).

The T98 method has not been tested in the nearshore with vertically separated sensors because (in particular) significant variation in vertical wave velocities may bias the T98 estimates. The T98 method also forms a Reynolds stress estimate averaged across the two sensors. Thus, estimating vertical Reynolds stress variation becomes problematic. This led ST01 to propose alternative differencing strategies and filtering methods resulting in Reynolds stress estimates at a single sensor. ST01 applied these methods to a near-bed vertical array of sensors (spanning from 0.4 to $7 \mathrm{~m}$ above the bed) deployed in 70-m water depth south of Martha's Vineyard, Massachusetts. Although many of the Reynolds stress estimation techniques worked well for low surface wave conditions (RMS velocities of $0.07 \mathrm{~m} \mathrm{~s}^{-1}$ ), only one technique worked well for more energetic surface wave conditions (RMS velocities of $0.12 \mathrm{~m} \mathrm{~s}^{-1}$ ). However, in general the wave velocities were weak relative to typical nearshore and surfzone conditions (RMS velocities never exceeded $0.22 \mathrm{~m} \mathrm{~s}^{-1}$ ). The ST01 method has not been applied in the much stronger wave velocities of the nearshore and surfzone. Here an extended method (denoted FW) is developed that combines the T98 and ST01 methods. The FW method reduces the wave bias more than the other two methods and still yields Reynolds stress estimates at a single sensor.

These three Reynolds stress estimation methods (described in section 2) are applied to a vertical array of acoustic Doppler velocimeter (ADV) observations with strong wave velocities made in 3.2-m mean water depth just offshore of the surfzone near Duck (section 3 ). During the 175-h-long experiment, the instrument site was seaward of the surfzone, and the alongshore current was largely wind driven. The T98 estimates of both $\overline{u^{\prime} w^{\prime}}$ and $\overline{v^{\prime} w^{\prime}}$ were clearly degraded by wave bias (section 4), largely resulting from the vertical variation of the vertical wave velocities. The ST01 and FW methods produce largely similar $\overline{u^{\prime} w^{\prime}}$ and $\overline{v^{\prime} w^{\prime}}$ estimates. However, the $\overline{v^{\prime} w^{\prime}}$ correlation between sensors is still low, contrary to the expected vertically uniform $\overline{v^{\prime} w^{\prime}}$, indicating additional problems with the estimates. A test of the integrated cospectrum (denoted ogive test) rejects the ST01 estimates roughly 50\% more than the FW estimates, and the FW estimates primarily are considered further.

In a predominantly wind-driven environment, a vertically uniform $\overline{v^{\prime} w^{\prime}}$ profile (i.e., a constant stress layer) is expected. The ogive test-passed FW $\overline{v^{\prime} w^{\prime}}$ estimates have magnitudes similar to the alongshore wind stress. The $\overline{v^{\prime} w^{\prime}}$ estimates at the three sensors are correlated

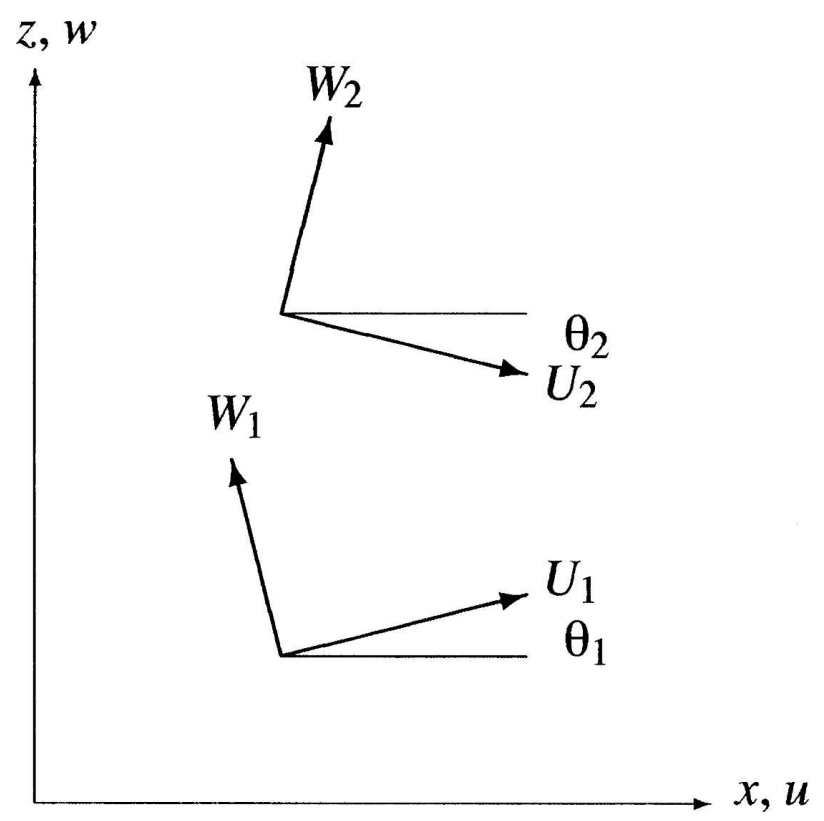

FIG. 1. Schematic illustrating the (2D) two-instrument geometry. The true coordinate system is given by $x$ and $z$ with velocities $u$ and $w$, respectively. The two instruments measure velocities $\left(U_{1}\right.$, $\left.W_{1}\right)$ and $\left(U_{2}, W_{2}\right)$ oriented at small angles $\theta_{1}$ and $\theta_{2}$, respectively, to the true coordinate system. The magnitudes of $\theta_{1}$ and $\theta_{2}$ are exaggerated for display purposes.

with slopes near one (section 5). The first EOF of FW $\overline{v^{\prime} w^{\prime}}$ (containing $73 \%$ of the variance) is vertically uniform, indicating that a constant stress layer is being observed. The demeaned first EOF amplitude of $\overline{v^{\prime} w^{\prime}}$ has a statistically significant nonzero correlation with both the wind stress and a parameterized quadratic bottom stress. The effect of wave bias on $\overline{v^{\prime} w^{\prime}}$ is explored for a larger range of wave and current conditions using empirical bottom stress parameterizations and derived wave bias terms (section 6), and the results are summarized in section 7 .

\section{Reynolds stress estimation methods}

\section{a. Background}

This analysis follows that of T98 and ST01. The coordinate system $(x, y$, and $z$, where $z=0$ at the bed) is defined so that $u, v$, and $w$ are the velocities in the offshore, alongshore, and upward direction, respectively (Fig. 1). Each velocity component is decomposed as (e.g., for $u$ )

$$
u=\bar{u}+\tilde{u}+u^{\prime}
$$

where the overbar, tilde, and primed quantities indicate the mean (time averaged), (irrotational) wave, and turbulent velocities, respectively. Wave and turbulent ve- 
locity fluctuations are assumed to be uncorrelated (e.g., $\overline{\tilde{u} u^{\prime}}=0$ ), and the waves and turbulence are assumed to be stationary over the sampling period (about $20 \mathrm{~min}$ ). The turbulent Reynolds stresses of primary interest here are $\overline{u^{\prime} w^{\prime}}$ and $\overline{v^{\prime} w^{\prime}}$. The following analysis is for $\overline{u^{\prime} w^{\prime}}$, but is easily adaptable to $\overline{v^{\prime} w^{\prime}}$.

In practice, the instrument coordinate systems are slightly rotated relative to the true coordinate system. Following ST01, the instrument coordinate system is assumed rotated in the two-dimensional (2D) $x, z$ plane by a small angle $\theta_{i}$ (Fig. 1). For reference, $3^{\circ}$ equals 0.05 rad. Assuming the sensors have a perfect response (no noise) and expanding to first order in $\theta_{1}$, the demeaned velocities measured by instrument 1 , denoted in capital letters, are

$$
\begin{aligned}
U_{1} & =\tilde{u}_{1}+u_{1}^{\prime}+\theta_{1}\left(\tilde{w}_{1}+w_{1}^{\prime}\right), \\
W_{1} & =\tilde{w}_{1}+w_{1}^{\prime}-\theta_{1}\left(\tilde{u}_{1}+u_{1}^{\prime}\right) .
\end{aligned}
$$

A tensor transformation (i.e., $U_{i}=a_{i j} u_{j}$; T98) allows for more general three-dimensional instrument rotations, but complicates the analysis.

As discussed in T98, direct evaluation of $\overline{U_{1} W_{1}}$ results in large wave bias terms (e.g., $\overline{\tilde{u}_{1} \tilde{w}_{1}}, \theta_{1} \overline{\tilde{u}_{1}^{2}}$, $\theta_{1} \overline{\tilde{w}_{1}^{2}}$ ) that swamp the Reynolds stress signal $\overline{u^{\prime} w^{\prime}}$. Thus, sensor velocity differencing strategies are pursued. Two existing Reynolds stress estimation methods (T98 and ST01) for use in the presence of orbital wave motions are examined as well as a new method that is an extension of the two. Forms for $\overline{u^{\prime} w^{\prime}}$ are presented here, for $\overline{v^{\prime} w^{\prime}}$ estimates, replace $U$ with $V$.

\section{b. T98 method}

The T98 estimation method is a two-sensor differencing method resulting in a Reynolds stress estimate averaged between the two sensors. Differencing the $U$ and $W$ velocities between sensors 1 and 2 yields

$$
\begin{aligned}
& \Delta U_{12}=U_{1}-U_{2}=\underbrace{\Delta \tilde{u}+\langle\theta\rangle \Delta \tilde{w}+\Delta \theta\langle\tilde{w}\rangle}_{\text {wave }}+\underbrace{u_{1}^{\prime}-u_{2}^{\prime}+\theta_{1} w_{1}^{\prime}-\theta_{2} w_{2}^{\prime}}_{\text {wave }}, \\
& \Delta W_{12}=W_{1}-W_{2}=\underbrace{\Delta \tilde{w}-\langle\theta\rangle \Delta \tilde{u}-\Delta \theta\langle\tilde{u}\rangle}_{\text {turb }}+\underbrace{w_{1}^{\prime}-w_{2}^{\prime}-\theta_{1} u_{1}^{\prime}+\theta_{2} u_{2}^{\prime}}_{\text {turb }},
\end{aligned}
$$

where the angle brackets $(\langle\rangle)$ represents an average over the sensors [e.g., $\langle\theta\rangle=\left(\theta_{1}+\theta_{2}\right) / 2$ ] and $\Delta$ represents a difference across sensors [e.g., $\left.\Delta \theta=\left(\theta_{1}-\theta_{2}\right)\right]$. By forming the product $1 / 2 \overline{\Delta U_{12} \Delta W_{12}}$, the resulting wave bias is [keeping terms only up to $O(\theta)$ or $O(\Delta \theta)$ ]

$$
\begin{aligned}
\frac{1}{2} \overline{\Delta U_{12} \Delta W_{12}} \mathrm{~W}= & \frac{1}{2}\left(\overline{\Delta \tilde{u} \Delta \tilde{w}}+\Delta \theta \overline{\langle\tilde{w}\rangle \Delta \tilde{w}}+\langle\theta\rangle \overline{(\Delta \tilde{w})^{2}}\right. \\
& -\langle\theta\rangle \overline{(\Delta \tilde{u})^{2}}-\Delta \theta \overline{(\tilde{u}\rangle \Delta \tilde{u})} .
\end{aligned}
$$

Following ST01, assuming that $\Delta \tilde{u}=\Delta z \partial \tilde{u} / \partial z$ (where $\Delta z$ is the vertical sensor separation), and representing the vertical variation of the wave-induced velocities to first order in $\sinh (k z)$ and $\cosh (k z)$ (where $k$ is the wavenumber) results in

$$
\Delta \tilde{u}=(\Delta z / z)(k z)^{2} \tilde{u}_{1}, \Delta \tilde{w}=(\Delta z / z) \tilde{w}_{1} .
$$

For vertically separated sensors in the nearshore $\Delta z / z$ is not small, but is of the order of 1 , and $k z<1$. Thus, differencing $U$ is more advantageous that differencing $W$. Expanding the T98 wave bias terms (3) results in (dropping the velocity subscripts)

$$
\frac{1}{2} \overline{\Delta U_{12} \Delta W_{12}} \mathrm{w}=\frac{\Delta z}{2 z}\left\{\frac{\Delta z}{z}(k z)^{2} \tilde{\tilde{u} \tilde{w}}+\Delta \theta\left[\overline{\tilde{w}^{2}}-(k z)^{2} \overline{\tilde{u}^{2}}\right]+\langle\theta\rangle \frac{\Delta z}{z}\left[\overline{\tilde{w}^{2}}-(k z)^{4} \overline{\tilde{u}}^{2}\right]\right\},
$$

and the reduction in wave bias relative to direct evaluation of $\bar{U}_{1} W_{1}$ is evident.

All components of $\overline{u_{i}^{\prime} u_{j}^{\prime}}$ are assumed to have the same order of magnitude. Thus, terms such as $\theta \overline{u_{1}^{\prime 2}}$ are much smaller than $\overline{u_{1}^{\prime} w_{1}^{\prime}}$. The resulting T98 turbulence terms (keeping terms without $\theta$ contributions) are

$$
\frac{1}{2}{\overline{\Delta U_{12} \Delta W_{12}}}^{\mathrm{t}}=\frac{1}{2}\left[\overline{u_{1}^{\prime} w_{1}^{\prime}}+\overline{u_{2}^{\prime} w_{2}^{\prime}}-\left(\overline{u_{1}^{\prime} w_{2}^{\prime}}+\overline{u_{2}^{\prime} w_{1}^{\prime}}\right)\right] .
$$

If turbulent velocities at the two sensors are uncorrelated (i.e., $\overline{u_{1}^{\prime} w_{2}^{\prime}}=0$ ), then (5) is an average of $\overline{u^{\prime} w^{\prime}}$ over 
the two sensors. However, in the nearshore and surfzone turbulence length scales scale with the water depth and some correlation is likely present, leading to a bias in (5). Additionally, ST01 found that in the non-wavedominated Hudson River $\Delta z / z$, should be greater than 5 for the turbulence to be considered uncorrelated. Thus, some turbulence bias is likely present with the T98 method in the nearshore.

\section{c. ST01 method}

Wave velocity magnitude variations or phase shifts can lead to large differenced wave velocities (i.e., $\Delta \tilde{u}$ ). To account for this, ST01 applied an adaptive filtering technique to map velocity from sensor 2 to sensor 1 before velocity differencing to reduce the wave bias. The filtering results in an adaptive filtered velocity $\hat{U}_{1}$ at sensor 1 , derived from $U_{2}$, is defined as

$$
\hat{U}_{1}(t)=\int_{-T / 2}^{T / 2} h(t-\tau) U_{2}(t) d \tau
$$

where $T$ is the filter convolution time based on the peak wave period and the filter weights $h$ are chosen to minimize least squares error $\left|\left[U_{1}(t)-\hat{U}_{1}(t)\right]^{2}\right|$. The details of the filtering are described in ST01. To assist in interpreting the filtering, a conceptual model for the results of the filtering is

$$
\hat{U}_{1}=F\left(U_{2}\right)=\tilde{u}_{1}+\varepsilon_{u} \Delta \tilde{u}+\tilde{n}_{u}+\delta_{u} u_{2}^{\prime},
$$

where $\varepsilon_{u}\left(\left|\varepsilon_{u}\right|<1\right)$ represents the reduction in wave bias, and $\tilde{n}_{u}$ is an unknown fraction of $\theta_{2} \tilde{w}_{2}$ that is passed by the filter and is considered noise. Filtering of the turbulent velocities is assumed to result in small $\delta_{u}$. The differenced velocity $\Delta \hat{U}_{12}$ is (neglecting $\tilde{n}_{u}$ )

$$
\Delta \hat{U}_{12}=U_{1}-\hat{U}_{1}=-\underbrace{\varepsilon_{u} \Delta \tilde{u}+\theta_{1} \tilde{w}_{1}}_{\text {wave }}+\underbrace{u_{1}^{\prime}-\delta_{u} u_{2}^{\prime}+\theta_{1} w_{1}^{\prime}}_{\text {turbulent }} .
$$

The ST01 Reynolds stress estimator is given by $\overline{\Delta \hat{U}_{12} W_{1}}$, and the wave bias is (to first order in $\theta$ )

$$
\overline{\Delta \hat{U}_{12} W_{1}} \mathrm{~W}=-\varepsilon_{u} \overline{\Delta \tilde{u} \tilde{w}_{1}}+\theta_{1} \varepsilon_{u}\left[\overline{\tilde{u}_{1} \Delta \tilde{u}}\right]-\theta_{1} \overline{\tilde{w}_{1}^{2}},
$$

which can be expressed as (dropping subscripts)

$$
\overline{\Delta \hat{U}_{12} W_{1}} \mathrm{w}=\varepsilon_{u}\left(\frac{\Delta z}{z}\right)\left[-(k z)^{2} \overline{\tilde{u} \tilde{w}}+\theta(k z)^{2} \overline{\tilde{u}^{2}}\right]-\theta \overline{\tilde{w}^{2}}
$$

and the filtering, through $\varepsilon_{u}$, reduces the wave bias. Note that in shallow water for normally incident monochromatic waves $\overline{\tilde{w}^{2}}=(k z)^{2} \overline{\tilde{u}}^{2}$. The turbulent stress terms are

$$
\overline{\Delta \hat{U}_{12} W_{1}}{ }^{\mathrm{t}}=\overline{u_{1}^{\prime} w_{1}^{\prime}}-\delta_{u} \overline{w_{1}^{\prime} u_{2}^{\prime}},
$$

resulting in a single-sensor Reynolds stress estimate, and with small $\delta_{u}$ the turbulence bias relative to T98 $[(5)]$ is removed.

\section{d. FW method}

In this method, the linear filtering is applied to both $U$ and $W$ followed by the T98 differencing strategy. Similar to the filtered $\hat{U}_{1}$ in (6), the filtered $\hat{W}_{1}$ is

$$
\hat{W}_{1}=F\left(W_{2}\right)=\tilde{w}_{1}+\varepsilon_{w} \Delta \tilde{w}+\tilde{n}_{w}+\delta_{w} w_{2}^{\prime},
$$

where $\varepsilon_{w}, \tilde{n}_{w}$, and $\delta_{w}$ are defined analogously to (6), resulting in differenced velocity $\Delta \hat{W}_{12}$ (neglecting the small unknown $\tilde{n}_{w}$ ),

$$
\Delta \hat{W}_{12}=W_{1}-\hat{W}_{1}=-\underbrace{\varepsilon_{w} \Delta \tilde{w}-\theta_{1} \tilde{u}_{1}}_{\text {wave }}+\underbrace{w_{1}^{\prime}-\delta_{w} w_{2}^{\prime}-\theta_{1} u_{1}^{\prime}}_{\text {turbulent }} .
$$

The FW Reynolds stress estimate $\overline{\Delta \hat{U}_{12} \Delta \hat{W}_{12}}$ is (to first order in $\theta$ )

$\overline{\Delta \hat{U}_{12} \Delta \hat{W}_{12}}{ }^{\mathrm{W}}=\varepsilon_{u} \varepsilon_{w}[\overline{\Delta \tilde{u} \Delta \tilde{w}}]+\varepsilon_{u} \theta_{1}\left[\overline{\tilde{u}_{1} \Delta \tilde{u}}\right]-\varepsilon_{w} \theta_{1}\left[\overline{\tilde{w}_{1} \Delta \tilde{w}}\right]$.

The FW wave bias terms in (12) are (dropping subscripts)

$$
\begin{aligned}
\overline{\Delta \hat{U}_{12} \Delta \hat{W}_{12}} \mathrm{w}= & \varepsilon_{u} \varepsilon_{w}\left(\frac{\Delta z}{z}\right)^{2}(k z)^{2} \overline{\tilde{u} \tilde{w}}+\theta_{1}\left(\frac{\Delta z}{z}\right) \\
& \times\left[\varepsilon_{u}(k z)^{2} \overline{\tilde{u}}^{2}-\varepsilon_{w} \overline{\tilde{w}^{2}}\right] .
\end{aligned}
$$

The second term in the ST01 wave bias [(9)] and the FW wave bias [(13)] are identical. The other FW terms are reduced relative to ST01 by $\varepsilon_{w}$ (or $\left.\Delta z / z\right)$. The turbulence stress terms are (without $\theta$ contributions) 


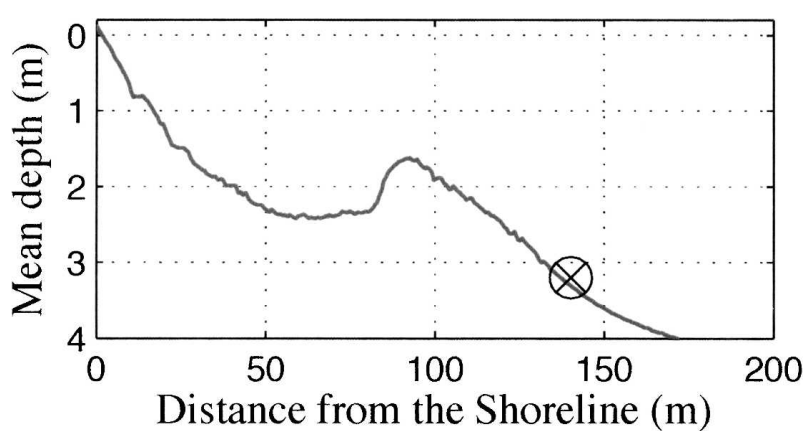

FIG. 2. Mean depth profile vs distance from the shoreline from a survey on 17 Sep 2002. The symbol marks the location of the tripod.

$$
{\overline{\Delta \hat{U}_{12} \Delta \hat{W}_{12}}}^{\mathrm{t}}=\overline{u_{1}^{\prime} w_{1}^{\prime}}-\left(\delta_{u} \overline{u_{2}^{\prime} w_{1}^{\prime}}+\delta_{w} \overline{u_{1}^{\prime} w_{2}^{\prime}}\right)+\delta_{u} \delta_{w} \overline{u_{2}^{\prime} w_{2}^{\prime}},
$$

and, similar to (10), with small $\delta_{u}$ and $\delta_{w}$ turbulence bias resulting from nonzero $\overline{u_{1}^{\prime} w_{2}^{\prime}}$ correlation is removed.

\section{Observations}

The Reynolds stress estimation techniques are tested with measurements collected during September 2002 off of a barrier island exposed to the Atlantic Ocean near Duck at the U.S. Army Corps of Engineers Field Research Facility (FRF). A vertical array of three Sontek ADVs was deployed on a tripod $140 \mathrm{~m}$ from shore in 3.2-m mean depth (Fig. 2), with a tide range of \pm 0.4 $\mathrm{m}$. The tripod was placed on the seabed and fixed in location (to prevent settling) by attachment to pipes jetted into the sand. At this location the beach slope is 0.02 , and offshore of the sandbar ( $90 \mathrm{~m}$ from shore) the bathymetry was highly alongshore uniform. The tripod orientation, pitch, and roll were determined by surveying the exposed tripod corners. The tripod tilt was consistent with the surveyed beach slope near the tripod. Tripod surveys were regularly performed during the experiment and showed no significant change in tripod orientation. Data were collected for 175 h (7.3 days) beginning at 1200 eastern standard time (EST) 18 September 2002.

The ADV has been both tested (Elgar et al. 2001) and used in turbulence studies (Trowbridge and Elgar $2001,2003)$ in the nearshore and surfzone regions. Velocities were measured at $12.5 \mathrm{~Hz}$ in hourly bursts of 24.8 min (18 572 data points). Data with velocity correlations $<0.7$ were rejected and interpolated over (Elgar et al. 2001). For the time periods considered, the number of rejected data points at any of the three ADVs for all bursts never exceeded $2.6 \%$ of the total data points, and on average were less $(0.4 \%, 0.8 \%$,

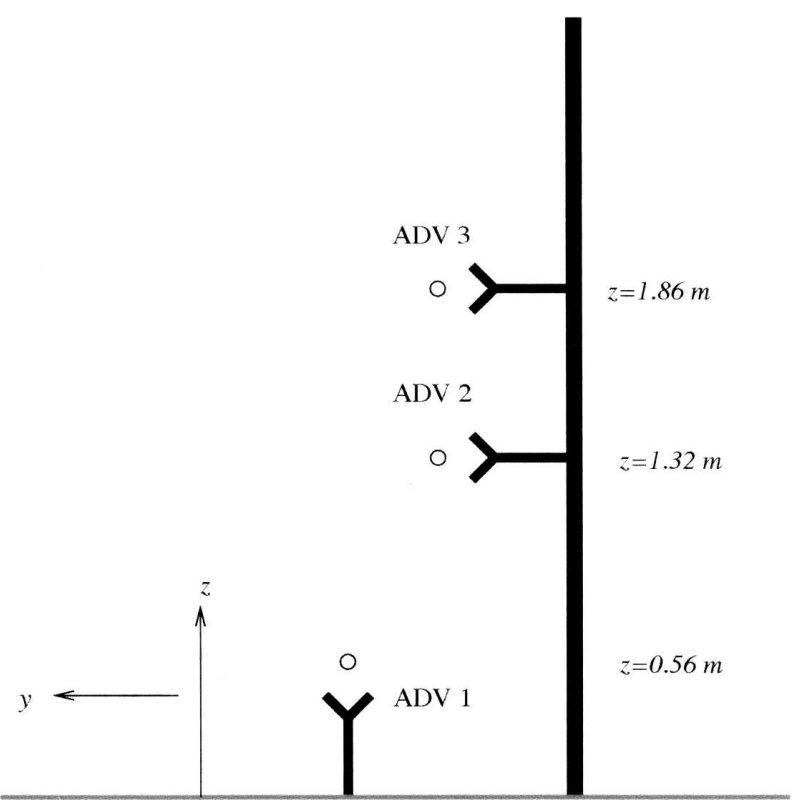

FIG. 3. Schematic of the ADV locations. The view is toward offshore $(+x)$, and the vertical $z$ and alongshore $y$ coordinates are indicated. ADV 1 is upward looking. The vertical location of the ADV sensing volumes (indicated by the small circle) is given. ADV 1 is offset approximately $0.56 \mathrm{~m}$ in the alongshore direction from the sensing volume of ADVs 2 and 3.

$1.6 \%)$. Both the mean and standard deviation of the data gap widths never exceeded five data points $(0.4 \mathrm{~s})$, and were typically about two data points. Occasionally data gaps of almost $2 \mathrm{~s}$ did occur, but were extremely infrequent.

The three ADVs (denoted ADV 1, 2, and 3) had sensing volumes at heights $0.56,1.32$, and $1.86 \mathrm{~m}$ above the bed, respectively (see Fig. 3). ADVs 2 and 3 were stacked, vertically mounted on the tripod mast and oriented sideways in the northward $(+y)$ alongshore direction. The bottom-most ADV 1 had an upwardlooking orientation, and its sensing volume was offset $0.56 \mathrm{~m}$ in the alongshore direction from ADVs 2 and 3 (Fig. 3). The $\Delta z / z$ between the adjacent instruments varies between 1.3 and 0.3 . Using the tripod survey information, the three components of ADV-measured velocity were transformed (rotated) into the FRF coordinate system with cross-shore $u$ (positive offshore), alongshore $v$ (positive northward), and vertical $w$ (positive upward) components. These rotated velocities are used throughout the manuscript.

Statistics of the mean flow field are given by burst means $(\bar{u}, \bar{v}, \bar{w})$ and standard deviations $\left(\sigma_{u}, \sigma_{v}, \sigma_{w}\right.$, where $\sigma_{u}=\left(\overline{\tilde{u}}^{2}\right)^{1 / 2}$ etc.) (Fig. 4). The mean cross-shore velocities $|\bar{u}|<0.1 \mathrm{~m} \mathrm{~s}^{-1}$ at all three ADVs are typically smaller than the alongshore current $\bar{v}$ (Figs. 4a,b). The mean vertical velocities $\bar{w}$ at all three ADVs are weak 

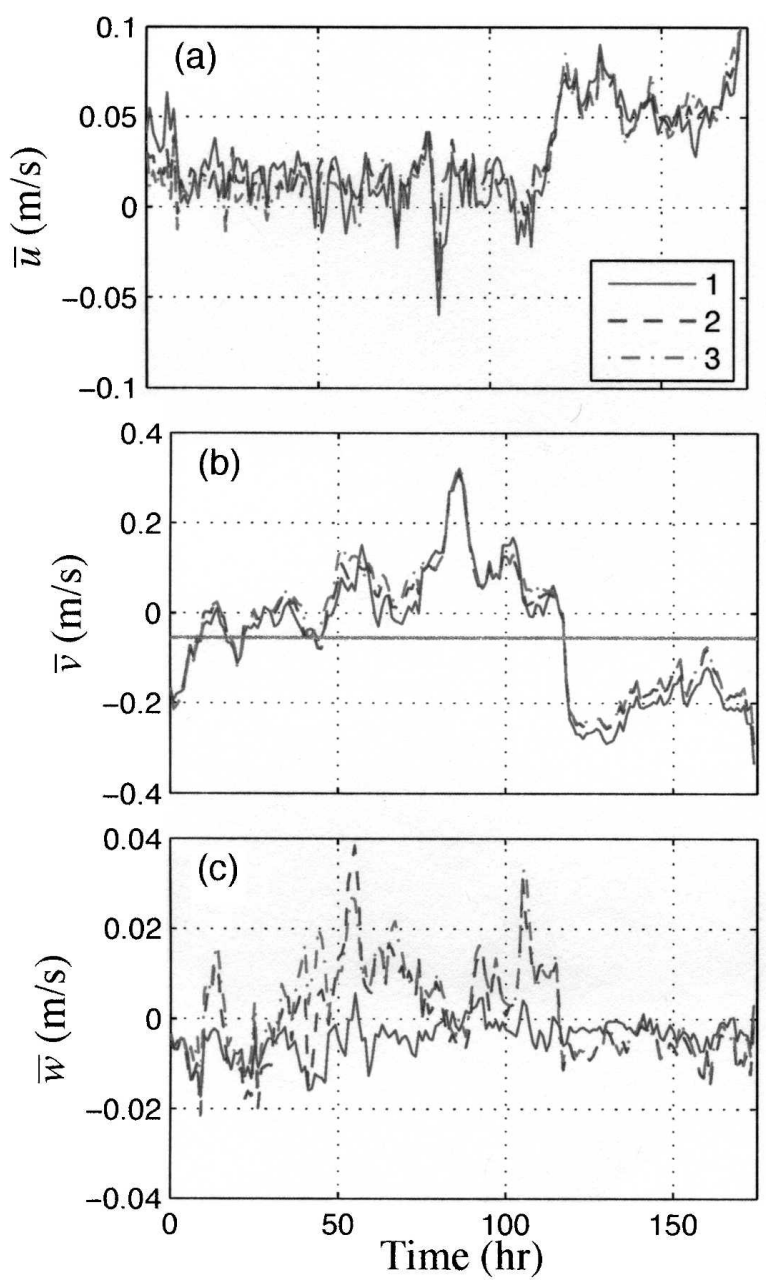
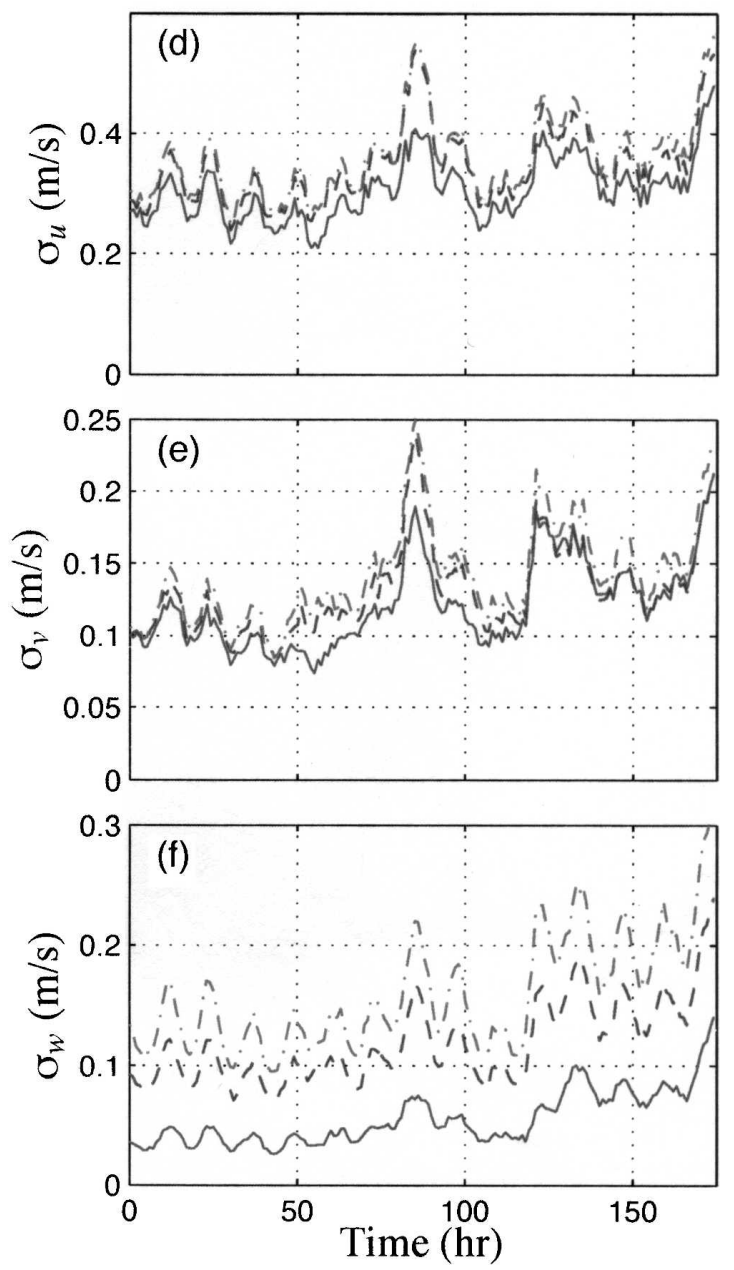

FIG. 4. Time series of mean (a) cross-shore $\bar{u}$, (b) alongshore $\bar{v}$, and (c) vertical $\bar{w}$ velocities and the standard deviations (d) $\sigma_{u}$, (e) $\sigma_{v}$, and (f) $\sigma_{w}$. The legend in (a) denotes the line types for the three ADVs. The horizontal gray line in (b) indicates the $\bar{v}=-0.07$ $\mathrm{m} \mathrm{s}^{-1}$ cutoff for conditions with instrument flow disturbance.

$\left(|\bar{w}|<0.04 \mathrm{~m} \mathrm{~s}^{-1}\right)$, with stronger vertical velocities near the surface (Fig. 4c), indicating that the coordinate transformation is reasonable. The significant wave height $H_{\text {sig }}$ varied between 0.5 and $1.1 \mathrm{~m}$ and the peak period was between 9 and $10 \mathrm{~s}$, resulting in typical $\mathrm{kz}$ of $0.08,0.2$, and 0.28 at the three ADVs. During the experiment, the tripod was seaward of the surfzone. This means that no depth-limited wave breaking (i.e., selfsimilar shoreward-propagating bore) occurred at the tripod. The orbital wave velocity standard deviation $\sigma_{u}$ varies by $0.2-0.5 \mathrm{~m} \mathrm{~s}^{-1}$, and $\sigma_{v}$ was about half of $\sigma_{u}$ (Figs. 4d,e). Thus, $\overline{u^{\prime} w^{\prime}}$ estimates have greater wave bias potential than $\overline{v^{\prime} w^{\prime}}$. The total horizontal orbital wave velocities $\sigma_{T}=\left(\sigma_{u}^{2}+\sigma_{v}^{2}\right)^{1 / 2}$ varied between 0.23 and $0.60 \mathrm{~m} \mathrm{~s}^{-1}$ and are strong relative to the $\sigma_{T}$ observed by ST01 (typically $<0.1 \mathrm{~m} \mathrm{~s}^{-1}$ ). The orbital vertical velocities $\left(\sigma_{w}\right)$ are also significant, and increase with height above the bed in a manner consistent with linear theory [i.e., $\left.\sigma_{w} \sim \sinh (k z) \sim k z\right]$. The semidiurnal variability in the wave velocities (e.g., $\sigma_{u}$ and $\sigma_{w}$ ) is due to tidal depth modulation of the wave shoaling (i.e., low tide results in larger waves).

With a northward-directed alongshore current (positive $\bar{v}$ ), the instruments measure in the lee of the tripod mast, and significant mast-induced flow disturbance is evident in the extremely elevated turbulence dissipation $\varepsilon$ (estimated using a technique similar to the one described in Trowbridge and Elgar 2001) relative to $\bar{v}$ of opposite sign (not shown). To reduce the effect of flow disturbance, the Reynolds stress analysis is restricted to cases where the highest instrument (ADV 3) has $\bar{v}<-0.07 \mathrm{~m} \mathrm{~s}^{-1}$ (gray horizontal line in Fig. 4b). This cutoff is chosen as a trade-off between minimizing flow disturbance and having sufficient data points for the analysis. Out of $175 \mathrm{~h}$ of data, $69 \mathrm{~h}$ satisfy this criterion and are included in the subsequent analysis. 
During these $69 \mathrm{~h}$, on average $|\bar{v}|<0.2 \mathrm{~m} \mathrm{~s}^{-1}$ and $\sigma_{T}=$ $0.33 \mathrm{~m} \mathrm{~s}^{-1}$.

The three Reynolds stress estimation methods-T98, ST01, and FW-are tested with these $69 \mathrm{~h}$ of data. T98 Reynold stress estimates averaged between sensors A and $\mathrm{B}$ are denoted as $\mathrm{T} 98 \mathrm{~A}(\mathrm{~B})$, or in the figure captions as $\overline{\Delta U_{\mathrm{AB}} \Delta W_{\mathrm{AB}}}$ (for $\overline{u^{\prime} w^{\prime}}$ ), where $\mathrm{A}$ and $\mathrm{B}$ are either 1,2 , or 3 . Note that the T98 A(B) and B(A) estimates are identical. The ST01 and FW method Reynold stress estimates at sensor A with adaptive-filtered velocities from sensor B are denoted as A(B). In the figure captions the two methods are denoted as $\overline{\Delta \hat{U}_{\mathrm{AB}} W_{\mathrm{A}}}$ and $\overline{\Delta \hat{U}_{\mathrm{AB}} \Delta \hat{W}_{\mathrm{AB}}}$, respectively.

For the ST01 and the FW methods, the adaptive filtering is performed as described in ST01, with a filter width of $9 \mathrm{~s}$ corresponding to the peak wave period. Changing the filter width does not change the results. The $\varepsilon_{u}, \varepsilon_{v}$, and $\varepsilon_{w}$ magnitudes for ADV pairs are estimated from a linear best-fit slope (for $\varepsilon_{u}$ ) between $\Delta \hat{U}_{12}$ in (7) and $\Delta U_{12}$ in (1). This assumes that the terms that do not involve $\Delta \tilde{u}$ are not correlated with $\Delta \tilde{u}$, and thus are noise in the fit. However, these $\varepsilon_{u, v, w}$ estimates are likely biased high because of nonzero correlations between, say, $\Delta \tilde{u}$ and the other terms. Similarly, $\varepsilon_{v}$ is the best-fit slope between $\Delta \hat{V}_{12}$ and $\Delta V_{12}$, and $\varepsilon_{w}$ is the best-fit slope between $\Delta \hat{W}_{12}$ and $\Delta W_{12}$.

During nonflow disturbance time periods, the $\varepsilon_{u}$ magnitude between ADVs 1and $2(\Delta z=0.76 \mathrm{~m})$ typically varies between 0.15 and 0.3 . The $\varepsilon_{u}$ between ADVs 2 and $3(\Delta z=0.54 \mathrm{~m})$ is typically larger, varying between 0.4 and 0.6 , resulting from the smaller sensor separation and thus smaller $\Delta \tilde{u}$. The $\varepsilon_{v}$ s are typically around 0.5 . The vertical orbital wave velocity varies the most (Fig. 4f), which has the potential to create bias through large $\Delta \tilde{w}$. The adaptive filtering of $\tilde{w}$ results in small $\varepsilon_{w}$, typically varying between 0.1 and 0.3 , and leads to significant reductions in wave bias in the FW method. There is an inverse relationship between $\varepsilon_{u}$ (and $\varepsilon_{v}, \varepsilon_{w}$ ) and $\sigma_{T}$ (and also $|\bar{v}|$ ).

Wind speed and direction measured $19.5 \mathrm{~m}$ above mean sea level at the end of the nearby FRF pier were used to estimate wind stress using the algorithm of Large and Pond (1981). No corrections were made for the likely significant effect of waves and white capping on the wind stress. The magnitude of the alongshore wind stress $\tau_{y}^{\mathrm{w}} / \rho$ is of the order of $10^{-4} \mathrm{~m}^{2} \mathrm{~s}^{-2}$ (Fig. 5). Because the tripod is seaward of the surfzone, the appropriate alongshore momentum balance is between the wind stress and bottom stress (Feddersen et al. 1998). For the 69 good hours of data, the alongshore wind stress is strongly related to a quadratic drag law for the bottom stress, that is,

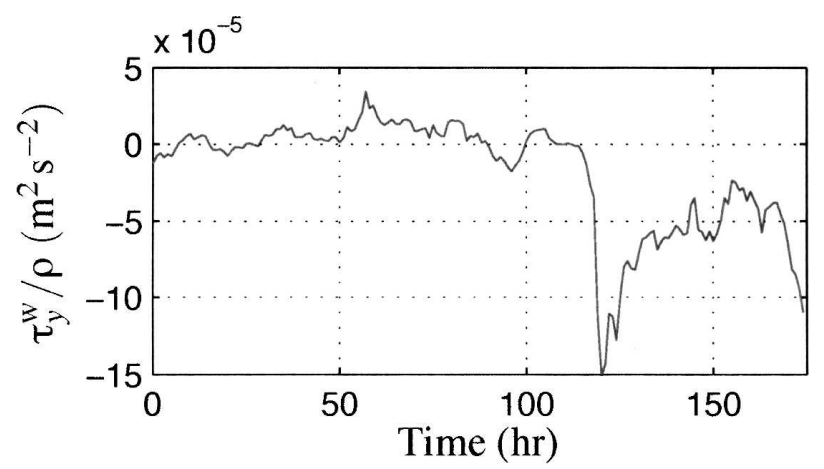

FIG. 5. Time series of alongshore wind stress $\tau_{y}^{\mathrm{w}} / \rho$. Positive $\tau_{y}^{\mathrm{w}} / \rho$ corresponds to northward wind stress.

$$
\tau_{y}^{\mathrm{w}} / \rho=c_{d} \overline{\mathbf{u} \mid v}
$$

where $c_{d}$ is a nondimensional drag coefficient and $|\mathbf{u}|$ is the horizontal velocity vector. The balance (14) is applied with ADV-1 velocity observations where the time average is over the 24.8-min burst. The skill of the balance (14) over the $69 \mathrm{~h}$ of data is high (correlation $r=$ 0.8 ), indicating that the alongshore current is largely wind driven. A wind-driven alongshore current has a depth-uniform $\overline{v^{\prime} w^{\prime}}$ profile. This concept will be used to test the quality of the $\overline{v^{\prime} w^{\prime}}$ estimates. Although no depth-limited wave breaking occurred at the tripod, occasional wind-induced white-capping wave breaking did occur during the later part of the experiment. The best-fit $c_{d}=0.7 \times 10^{-3}$ is consistent with the seaward of the surfzone momentum balances derived $c_{d}=10^{-3}$ at the same beach (Feddersen et al. 1998).

\section{Results}

\section{a. Intercomparison of methods}

As in ST01, the $\overline{u^{\prime} w^{\prime}}$ and $\overline{v^{\prime} w^{\prime}}$ estimates derived from the three methods are intercompared. The T98 $\overline{u^{\prime} w^{\prime}}$ estimates $(\overline{\Delta U \Delta W})$ between sensors 1(2) (Fig. 6a) and 3(2) (Fig. 6b) are an order of magnitude larger and a factor of 2 larger, respectively, and not are correlated with the ST01 $(\overrightarrow{\Delta \hat{U} W})$ and FW $(\overline{\Delta \hat{U} \Delta \hat{W}})$ estimates, indicating that the T98 $\overline{u^{\prime} w^{\prime}}$ estimates are contaminated by wave bias. Both the 1(2) and 3(2) ST01 and FW $\overline{u^{\prime} w^{\prime}}$ estimates are highly correlated with a one-to-one relationship (Figs. 6c,d), suggesting that either $\overline{u^{\prime} w^{\prime}}$ is accurately estimated or is contaminated by identical wave bias.

A similar pattern is seen in the $\overline{v^{\prime} w^{\prime}}$ intercomparison (Fig. 7). The T98 1(2) $\overline{v^{\prime} w^{\prime}}$ estimates are an order of magnitude larger than those of either the FW (Fig. 7a) or ST01 methods. The T98 3(2) estimates are a factor of 2-3 larger than the other two methods (Fig. 7b), but are 

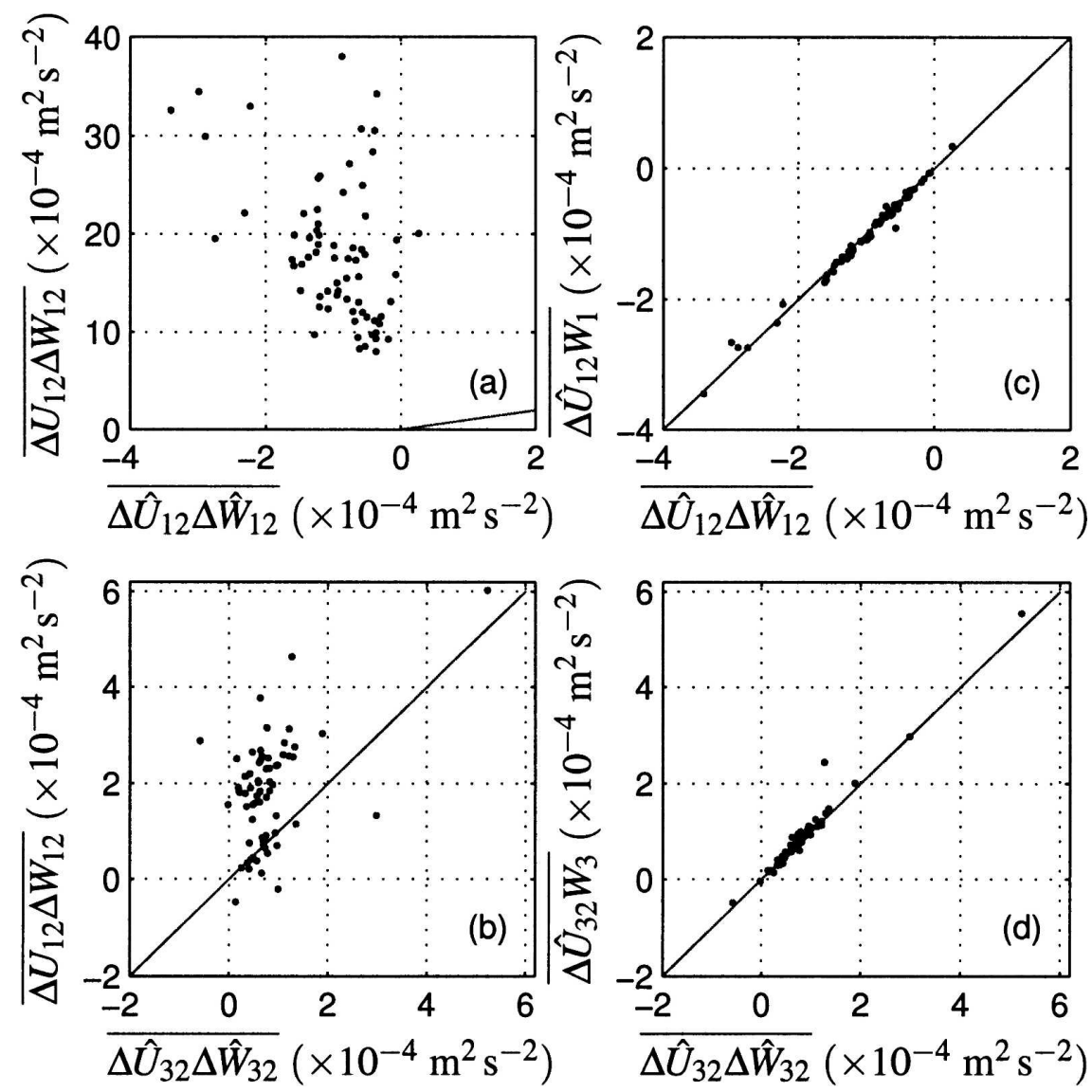

FIG. 6. Comparison of $\overline{u^{\prime} w^{\prime}}$ estimates from the three methods: T98 vs FW for sensors pairs: (a) 1(2) and (b) 3(2), and ST01 vs FW for sensors (c) 1(2) and (d) 3(2). There are a total of 69 data points, and the straight line shows the one-to-one relationship.

not uncorrelated. The T98 1(2) $\overline{u^{\prime} w^{\prime}}$ and $\overline{v^{\prime} w^{\prime}}$ estimates are worse than the 3(2) estimates because the larger $\Delta z$ between ADV 1 and 2 results in larger orbital wave velocity differences (Figs. 4d,e,f), particularly with $\sigma_{w}$. Using typical $\sigma_{w}$ and $\Delta \theta=0.05 \mathrm{rad}$, the largest T98 wave bias term for both $\overline{u^{\prime} w^{\prime}}$ and $\overline{v^{\prime} w^{\prime}}$ is the second term in (4) and equals $2.5 \times 10^{-4} \mathrm{~m}^{2} \mathrm{~s}^{-2}$, consistent with Figs. 6a,b and Figs. 7a,b. Use of the T98 method with vertically separated sensors in the nearshore is inappropriate.

The ST01 and FW $\overline{v^{\prime} w^{\prime}}$ are highly correlated with equal magnitudes (around $10^{-4} \mathrm{~m}^{2} \mathrm{~s}^{-2}$ ), similar to the wind stress magnitude (Fig. 5), suggesting that the $\overline{v^{\prime} w^{\prime}}$ estimates are not wave bias dominated. However, the similarity of the ST01 and FW $\overline{u^{\prime} w^{\prime}}$ does not necessarily imply that the Reynolds stress estimates are accurate. Indeed, with either method, $\overline{v^{\prime} w^{\prime}}$ estimates between either ADVs 1 and 2 or 2 and 3 have low correlations $(r$ $=0.2$ to $r=0.5$ ), contrary to the expected vertically uniform $\overline{v^{\prime} w^{\prime}}$ profile, indicating that additional problems with the ST01 and FW methods exist. Because $|\bar{v}|>|\bar{u}|$ but the ST01 and FW methods estimated $\left|\overline{u^{\prime} w^{\prime}}\right|>\left|\overline{v^{\prime} w^{\prime}}\right|$ suggests that the ST01 and FW $\overline{u^{\prime} w^{\prime}}$ are similarly wave dominated. The second term in (9) and (13) provides this mechanism.

\section{b. Ogive curve tests}

The cospectra of $u^{\prime} w^{\prime}$ and $v^{\prime} w^{\prime}$ derived from the three methods give insight into the quality of the Reynolds stress estimates. The nondimensional integrated cospectra $\mathrm{Og}_{u^{\prime} w^{\prime}}(f)$ (ogive curve) for $\overline{u^{\prime} w^{\prime}}$ is defined as

$$
\operatorname{Og}_{u^{\prime} w^{\prime}}(f)=\frac{\int^{f} \operatorname{Co}_{u^{\prime} w^{\prime}}(\hat{f}) d \hat{f}}{\overline{u^{\prime} w^{\prime}}},
$$

where $f$ is frequency and $\mathrm{Co}_{u^{\prime} w^{\prime}}$ is the $u^{\prime} w^{\prime}$ cospectrum. The $\overline{v^{\prime} w^{\prime}}$ ogive curve $\operatorname{Og}_{u^{\prime} w^{\prime}}(f)$ is defined similarly. Empirical forms for the ogive curves as a function of the nondimensional wavenumber $2 \pi f z / V$ ( $V$ is the along-stream velocity, and $2 \pi f / V$ is the apparent wavenumber of the turbulent eddies advected with steady 

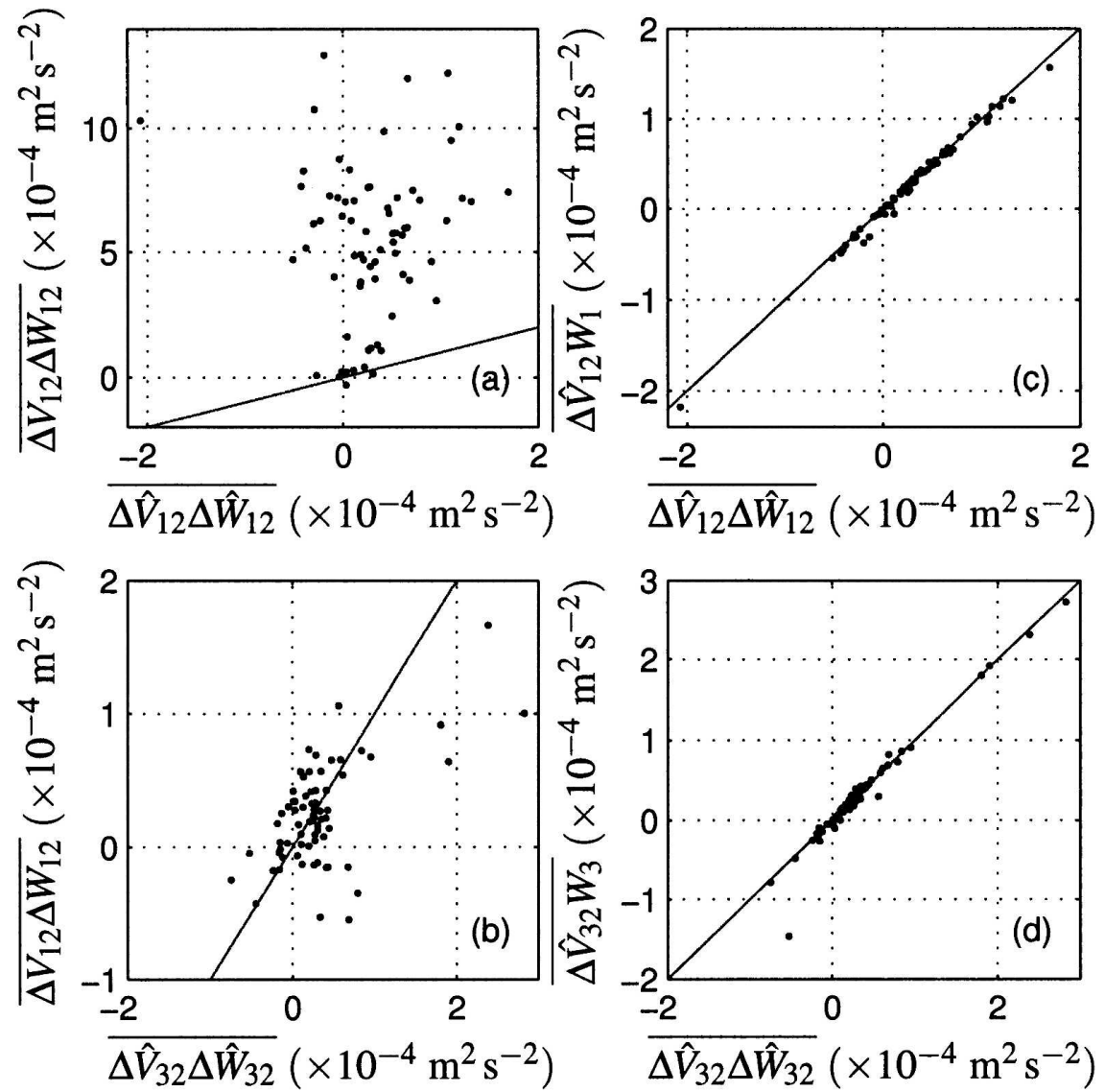

FIG. 7. Comparison of $\overline{v^{\prime} w^{\prime}}$ estimates from the three methods. T98 vs FW for sensors (a) 1(2) and (b) 3(2), and ST01 vs FW for sensors (c) 1(2) and (d) 3(2). There are a total of 69 data points, and the straight line shows the one-to-one relationship.

$V)$ were determined from measurements in the wall region of the atmospheric boundary layer (Kaimal et al. 1972). The majority of the contribution to the Reynolds stress occurs in the range $10^{-1}<2 \pi f z / V<10$. These empirical forms were consistent with cospectra obtained in a shallow-water tidal boundary layer without waves (Soulsby 1977). With orbital wave velocity motions, the wavenumber distribution changes from $2 \pi f / V$ (Lumley and Terray 1983). Nevertheless, as in ST01, ogive curves are presented as a function of $2 \pi f z / \bar{v}$ using the mean alongshore current in the place of $V$. ST01 presented ogive curves of their various Reynolds stress estimation methods on two case examples. The effect of wave bias was evident in the large steepness of the ogive curves.

Here ogive curves of $\overline{u^{\prime} w^{\prime}}$ and $\overline{v^{\prime} w^{\prime}}$ for the T98, ST01, and the FW methods are calculated (using 112-s-long records with $75 \%$ overlapping resulting in 100 degrees of freedom) for each of the $69 \mathrm{~h}$ of nonflow disturbance-contaminated data. Example ogive curves are plotted for the strongest $\bar{v}$ conditions (Fig. 8) at hour
124 with $H_{\text {sig }}=1.0 \mathrm{~m}$ and $\bar{v}=-0.28 \mathrm{~m} \mathrm{~s}^{-1}$. The T98 1(2) $\operatorname{Og}_{u^{\prime} w^{\prime}}(f)$ curve shows significant wave bias by the strong contribution over a narrow wavenumber range 1 $<2 \pi f z / \bar{v}<4$ (dash-dot curve in Fig. 8a), corresponding to the peak of the wave spectrum. This is characteristic of T98 $\overline{u^{\prime} w^{\prime}}$ and $\overline{v^{\prime} w^{\prime}}$ ogive curves in general (e.g., dashdot curve in Fig. 8b), and further confirms that the T98 method is inappropriate for use with vertically separated sensors in the nearshore. The ST01 and the FW methods often produce ogive curves similar to the Kaimal et al. (1972) empirical curves (cf. the solid and dashed curves to the black solid curves in Figs. 8a,b). However, the ST01 or the FW ogive curves also are at times clearly bad. For example, the ST01 3(2) $\mathrm{Og}_{u^{\prime} w^{\prime}}(f)$ (dashed curve in Fig. 8c) oscillates rapidly between -0.5 and 1.5 , whereas the FW $\mathrm{Og}_{u^{\prime} w^{\prime}}(f)$ (solid curve in Fig. 8c) generally follows the Kaimal et al. (1972) empirical form.

Ogive curves are used to reject bad ST01 or FW Reynolds stress estimates. The fit to the Kaimal et al. (1972) empirical curves cannot be used as a test to re- 

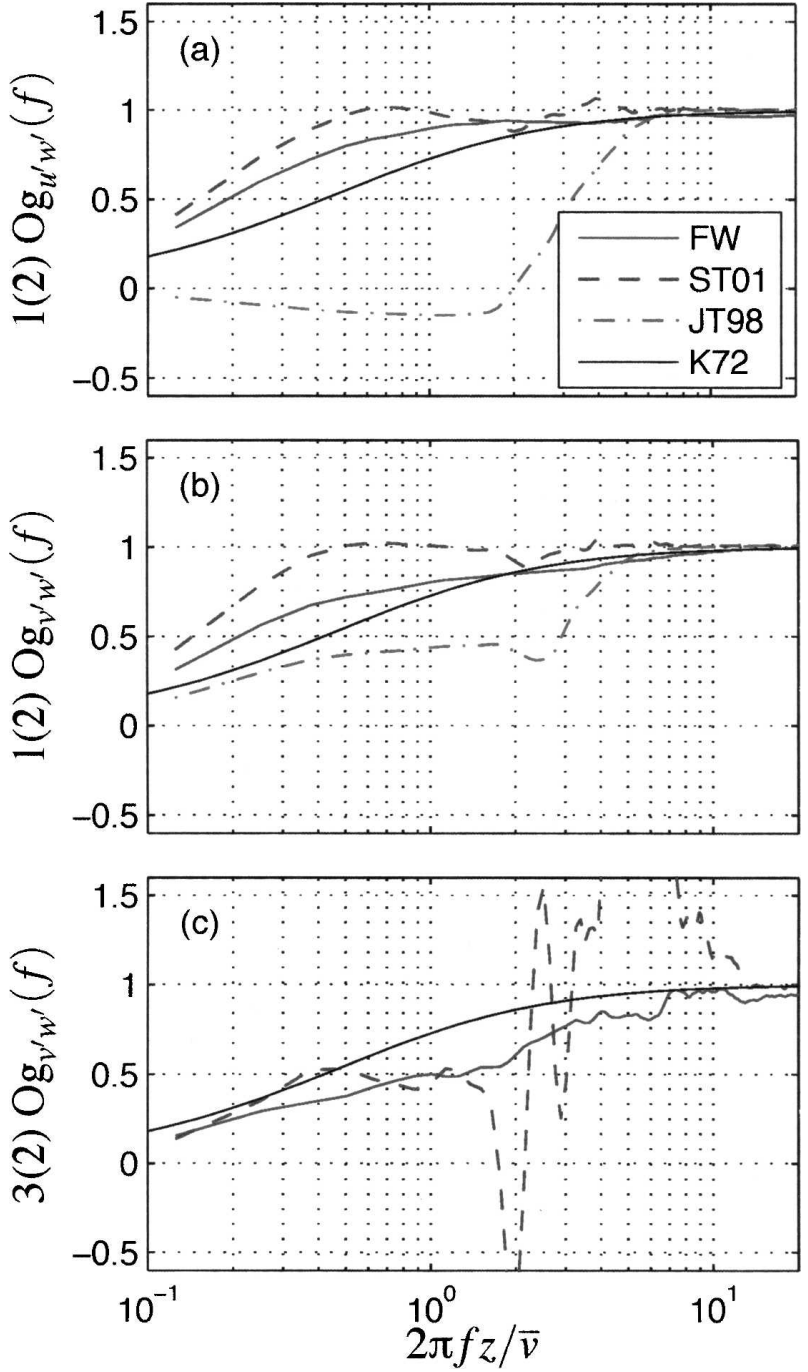

FIG. 8. Ogive curves vs nondimensional frequency $2 \pi f z / \bar{v}$ for (a) 1(2) $\mathrm{Og}_{u^{\prime} w^{\prime}}(f)$, (b) 1(2) $\mathrm{Og}_{v^{\prime} w^{\prime}}(f)$, and (c) 3(2) $\mathrm{Og}_{v^{\prime} w^{\prime}}(f)$ estimated with the FW (solid), ST01 (dashed), and T98 (dash-dot) methods at hour 124. The Kaimal et al. (1972) empirical curve (K72, black) is noted for reference. The conditions are $\bar{u}=0.07$ $\mathrm{m} \mathrm{s}^{-1}, \sigma_{u}=0.4 \mathrm{~m} \mathrm{~s}^{-1}, \bar{v}=-0.28 \mathrm{~m} \mathrm{~s}^{-1}, \sigma_{v}=0.2 \mathrm{~m} \mathrm{~s}^{-1}, \sigma_{w}=0.07$ $\mathrm{m} \mathrm{s}^{-1}$, and $H_{\text {sig }}=1.0 \mathrm{~m}$. At ADV $1,2 \pi f z / \bar{v}=1$ corresponds to $f$ $=0.08 \mathrm{~Hz}$.

ject bad Reynolds stress estimates because of the oscillatory wave velocities. Instead, Reynolds stress estimates are rejected if in the nondimensional wavenumber range $10^{-1}<2 \pi f z / \bar{v}<10, \operatorname{Og}(f)$ is not always in the range $-0.5<\operatorname{Og}(f)<1.6$. These heuristically selected limits maximize the number of Reynolds stress estimates that pass the test with the constraint of rejecting clearly bad estimates.

The percentage of FW Reynolds stress estimates that fail this test is between $23 \%$ and $35 \%$ (Table 1). The likelihood that the ogive test fails does not depend on
TABLE 1. Percentage of cases that failed the ogive curve test.

\begin{tabular}{lccccc}
\hline \hline & \multicolumn{2}{c}{$\overline{u^{\prime} w^{\prime}}$} & & \multicolumn{2}{c}{$\overline{v^{\prime} w^{\prime}}$} \\
\cline { 2 - 3 } \cline { 5 - 6 } & FW & ST01 & & FW & ST01 \\
\hline ADV 1(2) & 23 & 52 & & 20 & 46 \\
ADV 2(1) & 23 & 42 & & 35 & 54 \\
ADV 2(3) & 28 & 41 & & 29 & 49 \\
ADV 3(2) & 23 & 49 & 28 & 59 \\
\hline
\end{tabular}

$|\bar{v}|$ or $H_{\text {sig. }}$. The ST01 Reynolds stress estimates fail this test between $40 \%$ and $60 \%$ of the time (Table 1 ), which is about a factor of 1.5-2 greater than for FW. There is no indication of greater rejection likelihood for $\overline{u^{\prime} w^{\prime}}$ or $\overline{v^{\prime} w^{\prime}}$ with either method. The higher rejection rate of ST01 indicates that the FW method results in higherquality Reynolds stress estimates, and FW primarily will be discussed further.

\section{Vertical structure of Reynolds stress \\ a. Vertical structure of $\overline{u^{\prime} w^{\prime}}$}

Because of the weak mean cross-shore current $\bar{u}$ (Fig. $4 \mathrm{~b})$ and the lack of wave breaking, $\overline{u^{\prime} w^{\prime}}$ is expected to be smaller than $\overline{v^{\prime} w^{\prime}}$, with unknown vertical structure. The ogive test FW $\overline{u^{\prime} w^{\prime}}$ estimates have magnitudes of $O\left(10^{-4}\right) \mathrm{m}^{2} \mathrm{~s}^{-2}$, suggesting that these estimates are still wave bias contaminated and not useful for analyzing momentum or turbulence dynamics. The 1(2) and 2(1) $\overline{u^{\prime} w^{\prime}}$ estimates are highly correlated but are offset from the origin (Fig. 9a), indicating a wave bias proportional to $(\Delta z / z)$ [such as the second and third terms in (13)]. The 2(3) and 3(2) $\overline{u^{\prime} w^{\prime}}$ estimates are less affected by a $\Delta z / z$ wave bias term (Fig. 9b), because of the smaller $\Delta z / z$.

\section{b. Vertical structure of $\overline{v^{\prime} w^{\prime}}$}

Because $\bar{v}$ is stronger than $\bar{u}$ (Figs. 4a,b) (in fact only cases with $|\bar{v}|>0.07 \mathrm{~m} \mathrm{~s}^{-1}$ are considered, which is stronger than all $|\bar{u}|)$, which results in $\left|\overline{v^{\prime} w^{\prime}}\right|>\left|\overline{u^{\prime} w^{\prime}}\right|$, and because $\sigma_{v}$ is weaker than $\sigma_{u}$, the effect of wave bias is expected to be smaller for $\overline{v^{\prime} w^{\prime}}$ than for $\overline{u^{\prime} w^{\prime}}$. With no depth-limited wave breaking at the tripod and with the balance between wind stress and bottom stress, a depth-uniform $\overline{v^{\prime} w^{\prime}}$ is expected carrying the stress from the surface to the bed.

The ogive test-passed FW $\overline{v^{\prime} w^{\prime}}$ estimates $(\overline{\Delta \hat{U} \Delta \hat{W}})$ at the different ADVs are related to each other (Fig. 10) with similar magnitudes (approximately $10^{-4} \mathrm{~m}^{2} \mathrm{~s}^{-2}$ ) and generally high correlations ( $r$ between 0.5 and 0.7 ), with the exception of the 1(2) and 2(3) $\overline{v^{\prime} w^{\prime}}$ estimates $(r$ $=0.13$, Fig. 10a). Between 31 and 36 data points (out of 69) pass the ogive curve test simultaneously at both locations. The subset where the ogive test is simulta- 

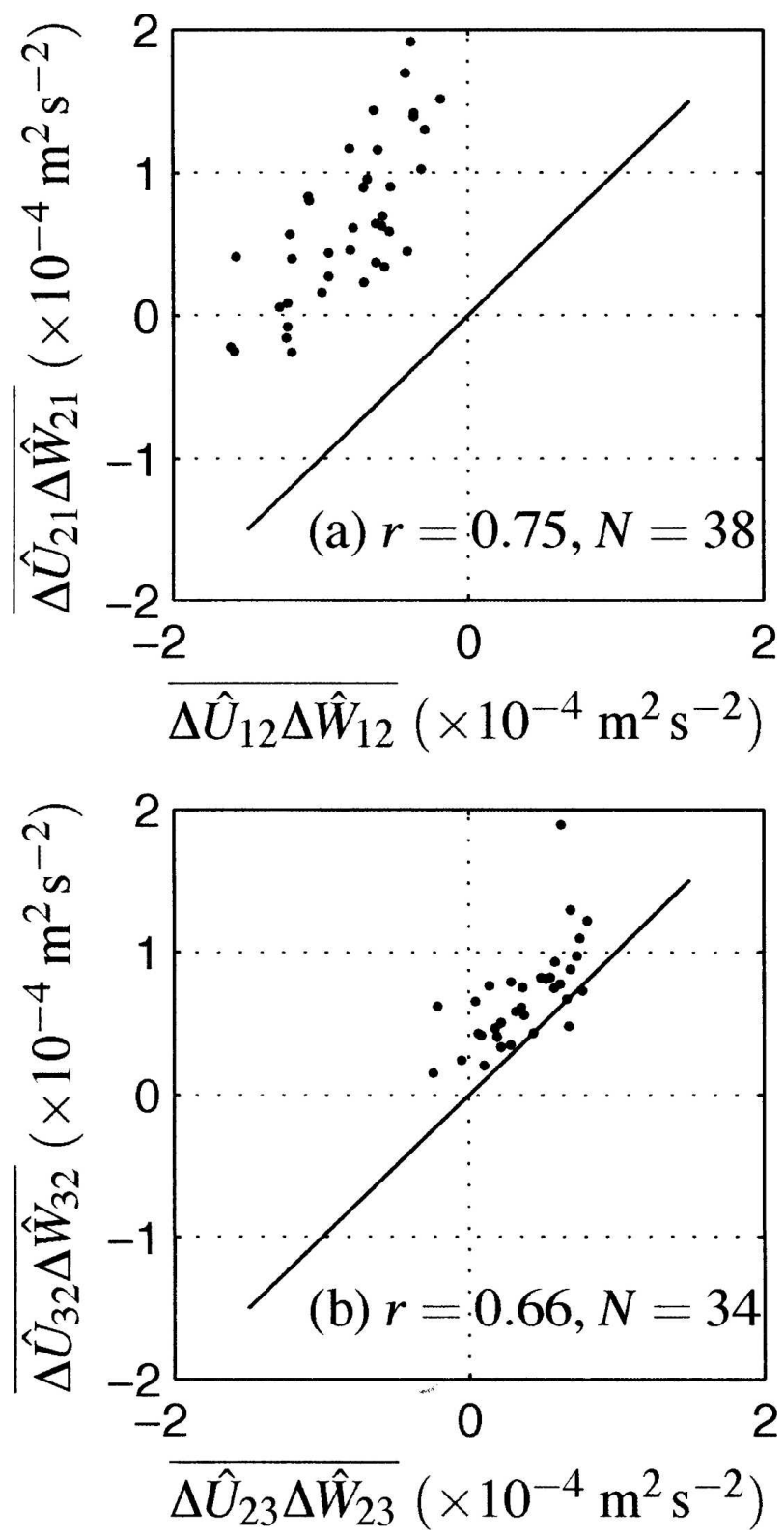

FIG. 9. Comparison of FW-estimated $\overline{u^{\prime} w^{\prime}}$ at adjacent sensors (a) 2(1) vs 1(2), and (b) 3(2) vs 2(3). The correlation $r$ and number of data points $N$ are given in the figure panels. The straight line shows a one-to-one relationship.

neously passed at all three locations [ADV 2 uses 2(3) because of the smaller $\Delta z / z]$ has 24 data points and the $\overline{v^{\prime} w^{\prime}}$ correlations improve [1(2) and 2(3) $r=0.38,2(3)$ and 3(2) $r=0.67$, and 1(2) and 3(2) $r=0.73$ ], indicating that a constant stress layer (i.e., $\overline{v^{\prime} w^{\prime}}$ independent of $z$ ) is observed for the nonflow disturbance-contaminated times.

For the ST01 method, between 13 and 21 data points (out of 69) pass the $\overline{v^{\prime} w^{\prime}}$ ogive curve test simultaneously at two ADV locations, which is about $50 \%$ less than the
FW method, and only 8 data points pass the test simultaneously at all three locations (relative to 24 for FW). The ogive-passed ST01 $\overline{v^{\prime} w^{\prime}}$ at the different ADVs also have a reduced correlation by $0.2-0.3$ relative to the FW estimates, further demonstrating that the ST01 method is not as robust as the FW method in these strong wave-dominated environments.

These 24 simultaneous FW $\overline{v^{\prime} w^{\prime}}$ estimates are demeaned and decomposed into EOFs,

$$
\overline{v^{\prime} w^{\prime}}\left(z_{i, t}\right)=\sum_{j=1}^{3} a_{j}(t) E_{j}\left(z_{i}\right)
$$

(e.g., Davis 1976), where $z_{i}$ is the height above the bed of the three ADVs $(i=1,2,3), a_{j}(t)$ is the $j$ th EOF amplitude $(j=1,2,3)$, and $E_{j}\left(z_{i}\right)$ is the EOF. The EOFs are the eigenvectors of the $\overline{v^{\prime} w^{\prime}}$ covariance matrix and are used to isolate spatially coherent patterns of variability. The first EOF of $\overline{v^{\prime} w^{\prime}}$ is nearly depth uniform (Fig. 11) and contains $73 \%$ of the $\overline{v^{\prime} w^{\prime}}$ variance, further indicating the presence of a constant stress layer. Given that $\bar{v}$ is largely wind forced, a constant stress layer is expected. Such a "classic" constant stress layer has never been observed in the coastal ocean in the presence of waves, and demonstrates the quality of the $\overline{v^{\prime} w^{\prime}}$ estimates.

\section{c. Relation of $\overline{v^{\prime} w^{\prime}}$ to wind stress and parameterized bottom stress}

If the balance between wind stress and bottom stress [(14)], with depth-uniform $\overline{v^{\prime} w^{\prime}}$, holds, then the depthuniform component of $\overline{v^{\prime} w^{\prime}}$ (EOF-1 amplitude of the FW $\overline{v^{\prime} w^{\prime}}$ estimates) should be related to the wind stress $\tau_{y}^{\mathrm{w}} / \rho$. These dynamics require that the wave Reynolds stress (i.e., $\tilde{\tilde{v} \tilde{w}}$ ) is zero (as expected for linear nonbreaking waves). The depth-uniform $\overline{v^{\prime} w^{\prime}}$ variability is given by $a_{1}(t) / \sqrt{3}$ (where $a_{1}$ is the first EOF amplitude) and is related to the demeaned wind stress (Fig. 12a) with correlation $r=0.36$, which is significant at the $95 \%$ level. Even with half the DOF (not all $24 \tau_{y}^{\mathrm{w}} / \rho$ are independent), the correlation is still significant to the $88 \%$ level. The best-fit slope is near one and the $y$ intercept is near zero, further indicating that the magnitude of the $\overline{v^{\prime} w^{\prime}}$ estimates is good. The first EOFderived $\overline{v^{\prime} w^{\prime}}$ is also related to the demeaned $-c_{d} \overline{\mathbf{u} \mid v}$ (Fig. 12b) with similar magnitudes and correlation $r=$ 0.38 . Recall that the wind stress and $c_{d} \overline{\mathbf{u} \mid v}$ were correlated with $r=0.8$ for the entire 69 -h non-flowcontaminated time period.

The relatively low correlations between $\overline{v^{\prime} w^{\prime}}$ and $-c_{d} \overline{|\mathbf{u}| v}$ are in contrast to the much higher correlation $(r=0.8)$ observed for a similar balance (but including alongshore wave forcing resulting from wave breaking) 

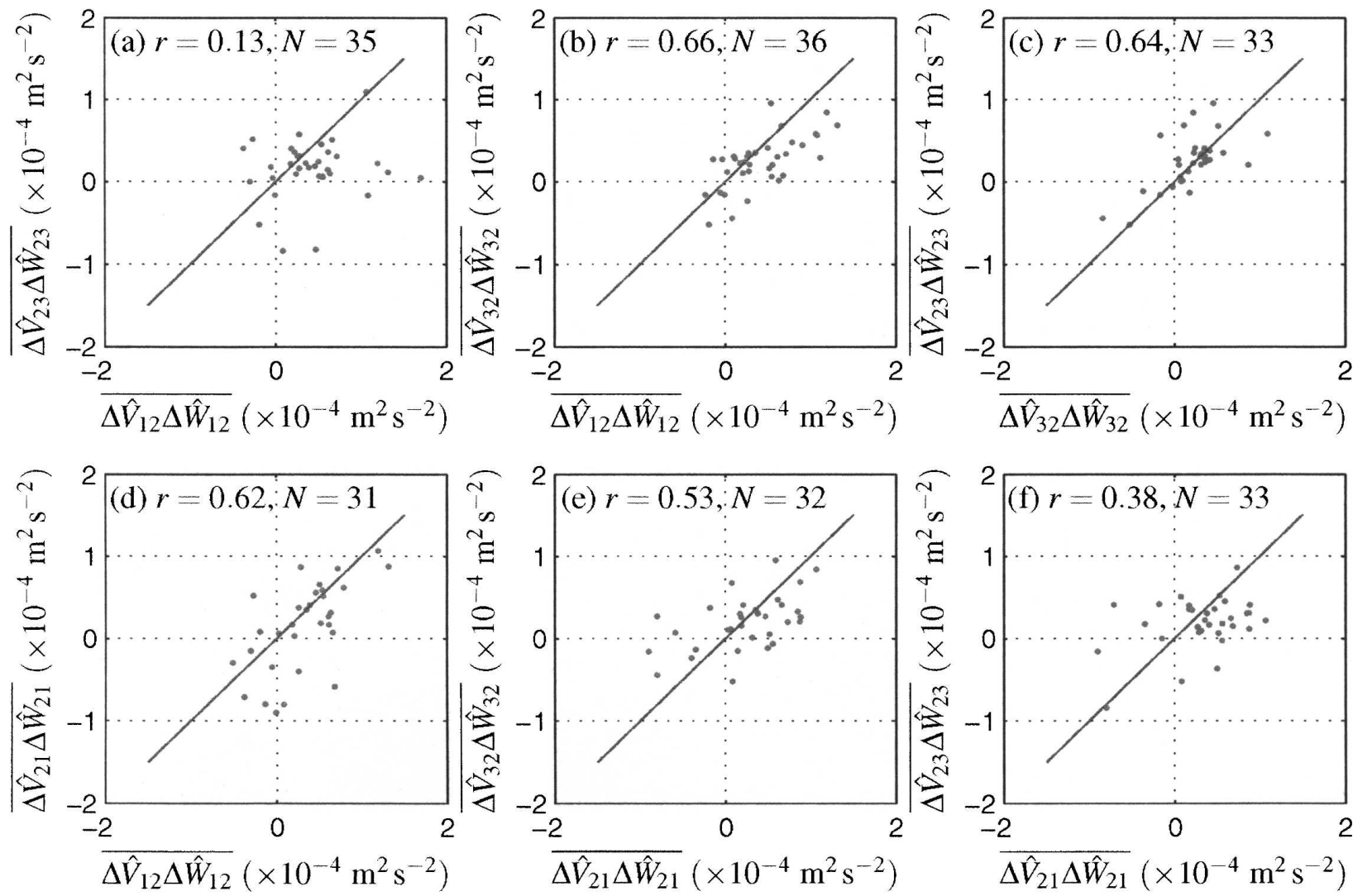

FIG. 10. Intersensor comparison of FW-estimated $\overline{v^{\prime} w^{\prime}}$ for all combinations of vertical location. The correlation $r$ and number of data points are given in the figure panels. The straight line shows the one-to-one relationship.

by Trowbridge and Elgar (2001) using the T98 method with horizontally separated current meters in $4.5-\mathrm{m}$ mean water depth. However, the dominant portion of the Trowbridge and Elgar (2001) signal occurred during periods of large breaking waves driving strong alongshore currents resulting in large $\overline{v^{\prime} w^{\prime}}$ of $O\left(10^{-3}\right) \mathrm{m}^{2} \mathrm{~s}^{-2}$, a factor of 10 larger than the $\overline{v^{\prime} w^{\prime}}$ observed here. Because the flows here are weaker, noise (either as lingering wave bias or instrument noise) affecting $\overline{v^{\prime} w^{\prime}}$ may reduce the correlation here. The wind stress is derived with an algorithm that does not account for the presence of waves, white capping, and proximity to the coast, which would have the tendency to degrade the $\overline{v^{\prime} w^{\prime}}$ and $\tau_{y}^{\mathrm{w}} / \rho$ correlation. Other processes, particularly the occasional white-capping wave breaking, which may act as a surface stress (Deigaard 1993), could also map into the $\overline{v^{\prime} w^{\prime}}$ EOF, reducing the correlation with $\tau_{y}^{\mathrm{w}} / \rho$

\section{Discussion}

The conditions observed during this deployment spanning 69 non-flow-contaminated hours are limited.
The wave height $H_{\text {sig }}$ typically varies between 0.5 and $1.0 \mathrm{~m}$, and the mean alongshore current was weak $\left(|\bar{v}| \leq 0.33 \mathrm{~m} \mathrm{~s}^{-1}\right)$. During these conditions, the FW method is often able to estimate quality $\overline{v^{\prime} w^{\prime}}$. It is not clear that for larger wave conditions the FW method would not also break down. To investigate this further, the size of the FW wave bias terms [(13)] relative to $\overline{v^{\prime} w^{\prime}}$ are examined over a range of alongshore current and orbital wave velocity conditions typical of the nearshore.

The fixed values of water depth of $h=3 \mathrm{~m}$, peak wave period $T=9 \mathrm{~s}$, instrument depth $z=h / 2$, instrument separation $(\Delta z / z)^{2}=1 / 2$, and instrument rotation $\theta=0.05 \mathrm{rad}\left(3^{\circ}\right)$ are chosen. Based on the adaptivefiltering fits, $\varepsilon_{v}=0.5$ and $\varepsilon_{w}=0.2$ are used. The total orbital wave velocity $\sigma_{T}$ and the mean alongshore current $\bar{v}$ are varied between 0.05 and $1 \mathrm{~m} \mathrm{~s}^{-1}$. The ratio $\sigma_{v} / \sigma_{u}=1 / 2$, typical of the observations here and of Duck in general (Feddersen et al. 2000), is used. The resulting $\sigma_{w}$ is calculated from linear theory using $h$ and $T$. The mean alongshore bottom stress and thus $\overline{v^{\prime} w^{\prime}}$ is estimated using the Wright and Thompson (1983) parameterization (as described in Feddersen et al. 2000) 


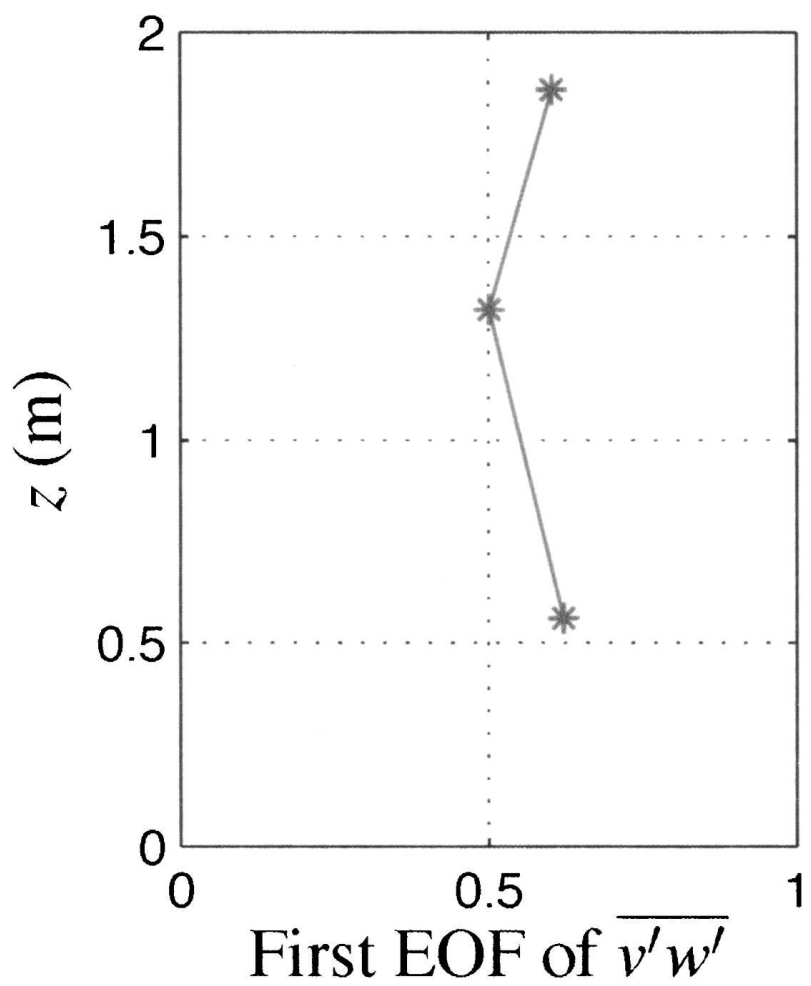

FIG. 11. The first EOF of FW-estimated $\overline{v^{\prime} w^{\prime}}, E_{1}\left(z_{i}\right)$ as a function of height above the bed $z$. This first EOF contains $73 \%$ of the variance.

$$
\overline{v^{\prime} w^{\prime}}=c_{d} \bar{v} \sigma_{T}\left[\alpha^{2}+\left(\bar{v} / \sigma_{T}\right)^{2}\right]^{1 / 2},
$$

where $\alpha=1.16$ is an empirical fit value, and the drag coefficient $c_{d}=1.5 \times 10^{-3}$ is chosen as a representative nearshore value (Feddersen et al. 1998).

With these parameters, the sizes of the $\mathrm{FW}$ wave bias terms for the range of $\bar{v}$ and $\sigma_{T}$ are calculated from (13).

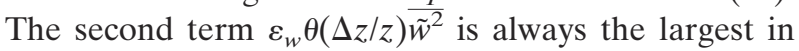
(13). Over the $\bar{v}$ and $\sigma_{T}$ parameter range, the ratio $\varepsilon_{w} \theta(\Delta z / z)\left(\overline{\tilde{w}^{2}} / \overline{v^{\prime} w^{\prime}}\right)$ varies over three orders of magnitude from $10^{-3}$ to 2.5 (Fig. 13) with a rapid decrease in the wave bias with stronger $\bar{v}$. For the typical example conditions observed here $\bar{v}=0.2 \mathrm{~m} \mathrm{~s}^{-1}$, and $\sigma_{T}=0.33$ $\mathrm{m} \mathrm{s}^{-1}$ (Fig. 8), the ratio is 0.18 (intersection of dark dashed curves in Fig. 13), further indicating that the wave bias has been sufficiently reduced to estimate $\overline{v^{\prime} w^{\prime}}$. There is a wide range of $\bar{v}$ and $\sigma_{T}$ where $\overline{v^{\prime} w^{\prime}}$ is not wave bias contaminated, and for $|\bar{v}|=0.5 \mathrm{~m} \mathrm{~s}^{-1}$, wave bias does not appear to be a concern.

For shallower water (reduced $\sigma_{w}$ resulting from linear theory) and larger $c_{d}$ associated with the surfzone (e.g., Feddersen et al. 1998), the wave bias is reduced even further relative to $\overline{v^{\prime} w^{\prime}}$. Using typical $\bar{u}=0.05 \mathrm{~m}$ $\mathrm{s}^{-1}$ and $\sigma_{T}=0.3 \mathrm{~m} \mathrm{~s}^{-1}$, the equivalent wave bias ratio for $\overline{u^{\prime} w^{\prime}}$ is 1.6. Except in strong undertow or rip current

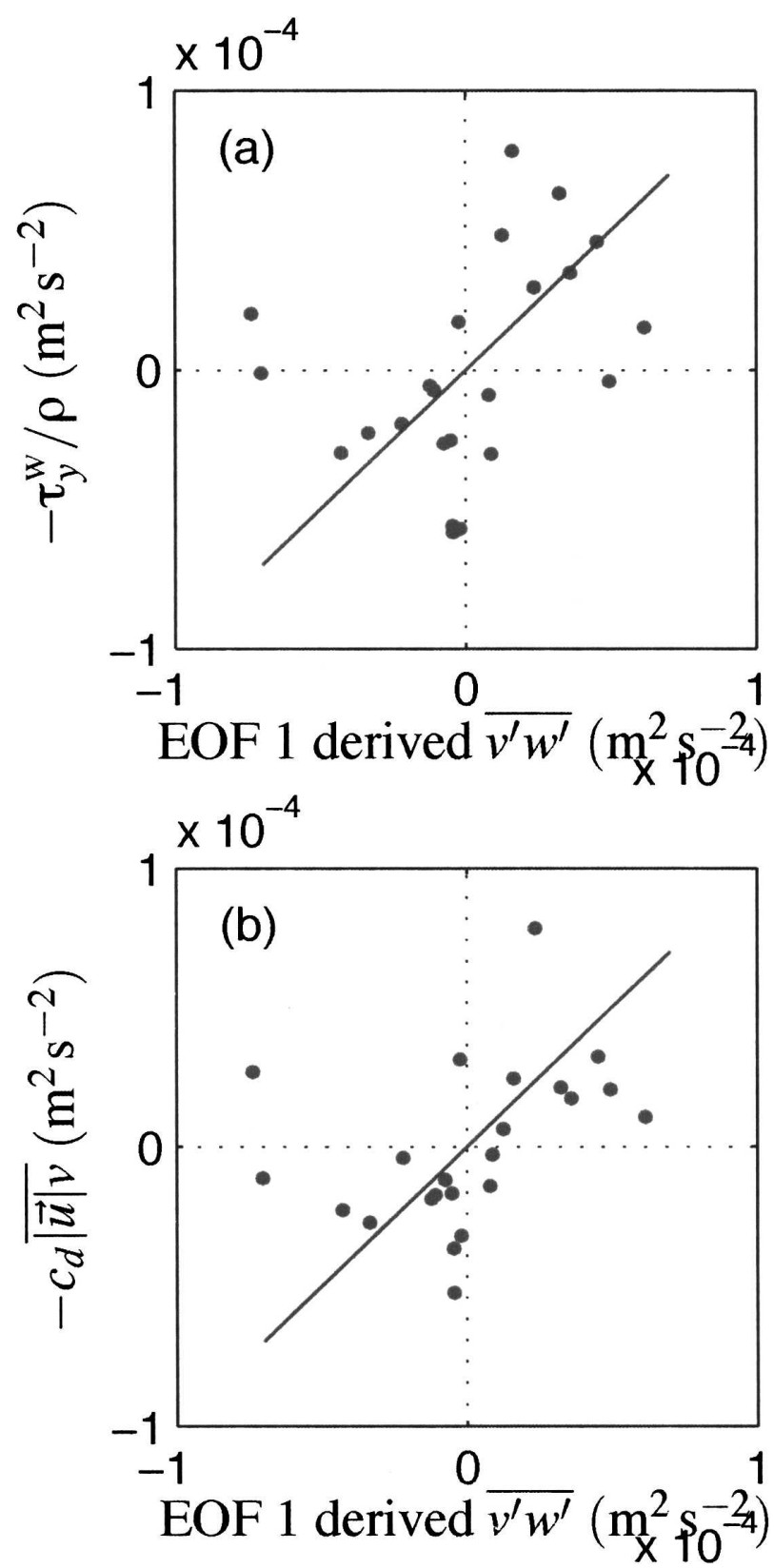

FIG. 12. (a) The demeaned $-\tau_{y}^{\mathrm{w}} / \rho$ and (b) $-c_{d} \overline{\mathbf{u} \mid v}$ vs the FW method EOF-1 amplitude-derived $\overline{v^{\prime} w^{\prime}}$. The best-fit $c_{d}=0.7 \times$ $10^{-3}$ from section 3 is used. The solid line represents a one-to-one relationship. The number of points is $N=23$ and the correlations (a) $r=0.36$ and (b) $r=0.38$ are significant at the $95 \%$ level.

conditions, it is unlikely that $\overline{u^{\prime} w^{\prime}}$ can be well estimated in the nearshore region.

\section{Summary}

Three different Reynolds stress $\left(\overline{u^{\prime} w^{\prime}}\right.$ and $\left.\overline{v^{\prime} w^{\prime}}\right)$ estimation methods, T98, ST01, and an extended method 


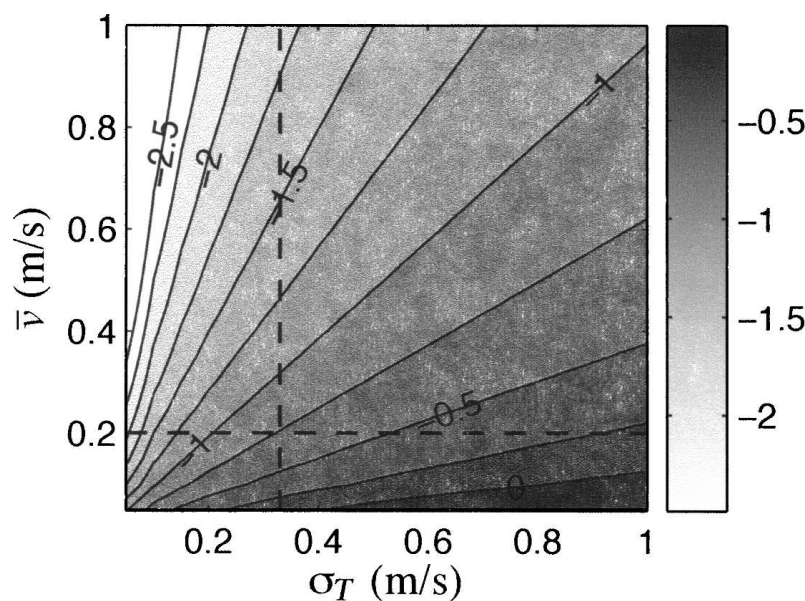

FIG. 13. Log10 contours of wave bias to $\overline{v^{\prime} w^{\prime}}$ ratio $\left(\varepsilon_{u} \varepsilon_{w}(\Delta z)\right.$ $\left.z)^{2} \theta_{1} \overline{\tilde{w}^{2}} / \overline{v^{\prime} w^{\prime}}\right)$ as a function of $\bar{v}$ and $\sigma_{T}$. The dark dashed lines show the typical $\bar{v}=0.2 \mathrm{~m} \mathrm{~s}^{-1}$ and $\sigma_{T}=0.33 \mathrm{~m} \mathrm{~s}^{-1}$ observed here.

$(\mathrm{FW})$, are testing using field observations collected in 3.2-m water depth off of a sandy ocean beach with strong wave orbital velocities. These methods involve differencing and filtering velocities from adjacent sensors, and are applied to a vertical array of acoustic Doppler velocimeters.

In contrast to situations where the T98 method has been successfully applied with horizontally separated sensors, the T98 method $\overline{u^{\prime} w^{\prime}}$ and $\overline{v^{\prime} w^{\prime}}$ estimates are dominated by wave bias resulting from the strong vertical variation in vertical orbital wave velocities. For a particular sensor pair, the ST01 and FW methods result in similar $\overline{u^{\prime} w^{\prime}}$ and $\overline{v^{\prime} w^{\prime}}$ estimates. However, similarity does not imply that the Reynolds stress estimates are accurate. A test based on the $u^{\prime} w^{\prime}$ (and $v^{\prime} w^{\prime}$ )integrated cospectra is used to reject bad ST01 and FW estimates. For adjacent sensor pairs, $23 \%-35 \%$ of the FW Reynolds stress estimates were rejected. The ST01 rejection rate was about $50 \%$ higher.

The vertical structure of the Reynolds stress estimates is used to further test the quality of the FW Reynolds stress estimates. The $\overline{u^{\prime} w^{\prime}}$ estimates between adjacent sensor pairs show clear evidence of wave bias. This is a result of (a) weak $\bar{u}$, which would make for small $\overline{u^{\prime} w^{\prime}}$ signal; and (b) strong cross-shore orbital velocities $\left(\sigma_{u}\right)$. Reliable estimates of $\overline{u^{\prime} w^{\prime}}$ in the nearshore therefore can be made only in much stronger undertow or rip current conditions.

The FW $\overline{v^{\prime} w^{\prime}}$ estimates at different sensors are all of a similar magnitude and are correlated with each other. This $\overline{v^{\prime} w^{\prime}}$ agreement is improved further when considering only cases when $\overline{v^{\prime} w^{\prime}}$ estimates exist at all three sensor locations simultaneously. The vertical structure of $\overline{v^{\prime} w^{\prime}}$ is examined with an EOF analysis. The first
EOF contains $73 \%$ of the variance and is depth uniform. A depth-uniform $\overline{v^{\prime} w^{\prime}}$ profile is expected for a purely wind-driven alongshore current. This is the first time the $\overline{v^{\prime} w^{\prime}}$ vertical structure in the wave-dominated nearshore has been measured. The first EOF-derived $\overline{v^{\prime} w^{\prime}}$ is related, albeit weakly, to both the alongshore wind stress and the quadratic drag law parameterized bottom stress.

The range of conditions observed was rather small. The effect of wave bias on the Reynolds stress is investigated over a larger mean alongshore current and orbital wave velocity range using empirical bottom stress formulations and the wave bias formulations given here. For the typical observed conditions $\left(\bar{v}=0.2 \mathrm{~m} \mathrm{~s}^{-1}\right.$ and $\sigma_{T}=0.33 \mathrm{~m} \mathrm{~s}^{-1}$ ), the $\mathrm{FW}$ wave bias term is about $18 \%$ of $\overline{v^{\prime} w^{\prime}}$. The wave bias to $\overline{v^{\prime} w^{\prime}}$ ratio decreases rapidly with larger $\bar{v}$. Estimating $\overline{v^{\prime} w^{\prime}}$ in the shallower water of the surfzone would result in a reduced wave bias.

Acknowledgments. Funded by NSF, ONR, and NOPP. The Field Research Facility, Coastal Engineering Research Center, in Duck, North Carolina, provided logistical support for the tripod deployment, bathymetric surveys, and wind, wave, and tide data. John Trowbridge and Janet Fredericks assisted with the fieldwork. Steve Henderson, R. T. Guza, and John Trowbridge provided valuable feedback.

\section{REFERENCES}

Davis, R. E., 1976: Predictability of sea surface temperature and sea level pressure anomalies over the North Pacific Ocean. $J$. Phys. Oceanogr., 6, 249-266.

Deigaard, R., 1993: A note on the three-dimensional shear stress distribution in a surfzone. Coastal Eng., 20, 156-171.

Elgar, S., R. T. Guza, and B. Raubenheimer, 2001: Current meter performance in the surf zone. J. Atmos. Oceanic Technol., 18, 1735-1746.

Feddersen, F., R. T. Guza, S. Elgar, and T. H. C. Herbers, 1998: Alongshore momentum balances in the nearshore. J. Geophys. Res., 103, 15 667-15 676.

,,--- , and -2000 : Velocity moments in alongshore bottom stress parameterizations. J. Geophys. Res., 105, 86738686.

Grant, W. D., and O. S. Madsen, 1986: The continental shelf bottom boundary layer. Annu. Rev. Fluid Mech., 18, 265-306.

Kaimal, J. C., J. C. Wyngaard, Y. Izumi, and O. R. Cote, 1972: Spectral characteristics of surface layer turbulence. Quart. J. Roy. Meteor. Soc., 98, 563-589.

Large, W. G., and S. Pond, 1981: Open ocean momentum flux measurements in moderate to strong winds. J. Phys. Oceanogr., 11, 324-336.

Lumley, J. L., and E. A. Terray, 1983: Kinematics of turbulence convected by a random wave field. J. Phys. Oceanogr., 13, 2000-2007.

Shaw, W. J., and J. H. Trowbridge, 2001: The measurement of 
near-bottom turbulent fluxes in the presence of energetic wave motions. J. Atmos. Oceanic Technol., 18, 1540-1557.

Soulsby, R. L., 1977: Similarity scaling of turbulence spectra in marine and atmospheric boundary layers. J. Phys. Oceanogr., 7, 934-937.

Trowbridge, J. H., 1998: On a technique for measurement of turbulent shear stress in the presence of surface waves. J. Atmos. Oceanic Technol., 15, 290-298.
_ and Y. C. Agrawal, 1995: Glimpses of a wave boundary layer. J. Geophys. Res., 100, 20 729-20 743.

_, and S. Elgar, 2001: Turbulence measurements in the surf zone. J. Phys. Oceanogr., 31, 2403-2417.

_ and - 2003: Spatial scales of stress-carrying nearshore turbulence. J. Phys. Oceanogr., 33, 1122-1128.

Wright, D. G., and K. R. Thompson, 1983: Time-averaged forms of the nonlinear stress law. J. Phys. Oceanogr., 13, 341-346. 